\title{
Genital herpes: the undiscovered pandemic
}

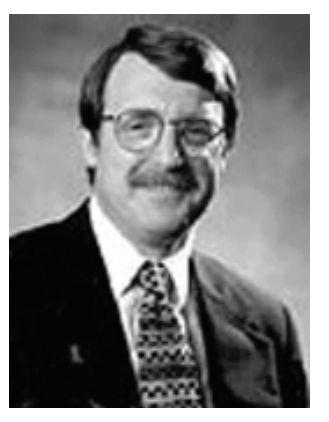

Brian Conway

University of British Columbia, Downtown ID Clinic, 201-1200 Burrard Street, Vancouver, BC V6Z2C7, Canada Tel.: + 16046426429 ; Fax: +1 6046426419 ; bconway@interchange.ubc.ca

future, part of $\mathrm{fsg}$

\section{'As there are over \\ 50 million Americans \\ living with genital herpes ... to refer to (this condition) as a pandemic is clearly justified.'}

The word 'pandemic' is used easily in the medical literature (perhaps more so in literature directed to the general public) to describe a condition so widespread as to have a direct and measurable impact on the health of the population of the entire world. Wikipedia tells us that a pandemic is a global epidemic or an outbreak of an infectious disease that affects people or animals over an extensive geographical area (from the Greek words 'pan', all, and 'demos', people). We refer to a pandemic of HBV, HIV, chlamydia and even human Papillomavirus (HPV) infection, the latter being increasingly mentioned because of the availability of a vaccination. It is hoped that the targeting of this vaccination to young girls before the onset of sexual activity will play an important part in the control of HPV-associated cervical malignancy. This is especially true if we compare it with the prevalence of HBV $(400,000)$, HIV $(560,000)$ and chlamydia (2 million), each infection being called a pandemic at various times [1].

As there are over 50 million Americans living with genital herpes, herpes simplex virus (HSV)-1 and -2 infections deserve at least equal recognition, if only based on the shear magnitude of the problem that may face healthcare practitioners [1]. Almost 1\% of North Americans will become newly infected with one of the agents of genital herpes over the next year [2].

In our own province of British Columbia, a survey conducted in 1999 showed that $17.3 \%$ of pregnant women had positive serologic tests for HSV-2 antibodies and were thus chronically infected with the virus and able to transmit it to their sexual partners, or even to their children during labor [3]. These data clearly show us that to refer to genital herpes as a pandemic is clearly justified. This is not only based on the prevalence data, but also on the fact that it affects a broad cross section of the population and is not limited to certain individuals or groups that are easily identified and targeted for intervention.

So why are physicians and other healthcare practitioners not tackling a problem of this magnitude more urgently and comprehensively? I believe that there are at least four reasons why this is so:

- Lack of perception of the actual magnitude of the problem

- Lack of a clear understanding of the way in which the problem has developed

- Lack of knowledge about the tools available to us to intervene

- Lack of understanding about the type of commitment that it would be feasible to make within a busy primary healthcare environment

Many physicians have a hard time believing that $20 \%$ of the population is infected [4], since they do not see that $20 \%$ of their time is spent dealing with this condition. In fact, only $10 \%$ of infected individuals actually seek care for the condition [4]. Unless physicians seek out cases on an active basis, they may only spend $2 \%$ or less of their time dealing with the selfdeclared cases, and perceive they have dealt with the entire scope of the problem as it relates to their practice.

'Unless physicians seek out cases on an active basis, they may only spend $2 \%$ or less of their time dealing with the self-declared cases.'

The root cause of this discordance is based on the key role of asymptomatic viral shedding in the pathogenesis of the disease and its transmission. This is defined as the presence of culturable HSV in the anogenital region without any clinically detectable lesions or symptoms [4-6]. Although the frequency of shedding is most frequent in the first year following infection, it persists throughout the lifetime of the subject, being documented on $2-3 \%$ of days (or an average of 1 day/month) [6,7]. If one uses more 
sensitive molecular tests, fourfold higher rates of shedding are observed [8]. Although the true clinical significance of this higher rate is unclear, to say that all infected individuals are infectious 1 day each month is a truly minimal estimate. Epidemiologic data initially published in 1992 show that $70 \%$ of transmission of new infections occurs during periods of asymptomatic viral shedding [5].

Based on these facts, we can now develop a clear model of how the prevalence of genital herpes has increased to its current level. A given person would become infected with genital herpes and then become asymptomatic, either as a result of host immunologic control or pharmacologic intervention. Over the course of the subsequent year, they would go on to be well the majority of the time, but infectious a few days a month even in the absence of symptoms. In the scenario of a heterosexual relationship, over $35 \%$ of uninfected women would acquire genital herpes over the course of a year, especially if they do not carry antibodies to HSV-1 [5]. If they do, the rate decreases to about $10 \%$, a rate still high enough to sustain a pandemic of the magnitude that we are observing [5]. Even female-to-male transmission; occurring at a rate of $5 \%$ or less/year, will contribute significantly to the maintenance of the disease burden in the population [5]. If we add to this the observation that $30 \%$ or more of the new genital herpes infections are never symptomatic at any point [8], we have a truly silent pandemic, incorporating many individuals who never knew they were infected passing on the virus to others, who may not find out they are infected until they pass it on to a subsequent partner.

The key to the initial control of the pandemic is to identify the opportunities for transmission and intervene in such a way as to interrupt them. Short of targeting the entire population (which is impractical and probably not a good investment of healthcare resources), a more reasonable approach might be to target the situations carrying the highest risk of transmission [5,9]. These include:

- Female partners of infected males

- Female or male partners of newly infected individuals

- Relationships where more frequent sexual contact occurs

- Newer relationships, such as those of less than 2 years' duration
And what form should the intervention take? Corey and colleagues led an important study to address this issue, building on the fact that daily suppressive therapy with an antiviral agent has been shown to markedly reduce rates of HSV-2 shedding in the genital tract [9]. This international, randomized, double-blind, placebocontrolled study was designed to evaluate whether suppressive therapy with valacyclovir would reduce the risk of transmission of genital herpes in couples discordant for HSV-2. Source (or infected) partners were randomized to receive valacyclovir $500 \mathrm{mg}$ once daily or placebo for 8 months. Open-label valacyclovir therapy (at a dose and duration consistent with current clinical guidelines) was provided for any clinical recurrence. Susceptible partners were evaluated monthly for genital herpes. At each monthly visit, all couples were counseled to practice safer sex, including the use of condoms. The study population was made up of immunocompetent heterosexual adults in a stable monogamous relationship, the source partner having approximately five episodes of genital herpes/year.

'The key to the initial control of the pandemic is to identify the opportunities for transmission and intervene in such a way as to interrupt them.

Over the course of the Corey study [9], 3.6\% of the placebo group (27 out of 741) experienced a seroconversion for genital herpes, as compared with $1.9 \%(14 / 743)$ couples $(\mathrm{p}=0.04)$ where the source partner was receiving valacyclovir. If only symptomatic infections were considered, these figures were $2.2 \%$ (16 out of 741$)$ and $0.5 \%$ (four out of 743 ), $p=0.008$. The benefit of valacyclovir is not only to reduce the incidence of transmission, but also to reduce the severity of the symptoms associated with acute infection, to the point where over $80 \%$ of new infections were asymptomatic (as compared with only $40 \%$ in the placebo group).

At first glance, this seems to suggest that the key to controlling the genital herpes pandemic would be to proceed with an aggressive case-finding strategy and place all infected individuals on valacyclovir. This will interrupt new transmissions and, over time, lead to a reduction in the prevalence of disease, much as the implementation of universal immunization for HBV is leading to a significant alteration in the epidemiology of this chronic viral infection. 
An additional piece of data from the Corey study sheds light on how this may play out, from a practical perspective [9]. Despite all the attempts at instruction on risk reduction for transmission, $37 \%$ of the couples enrolled in the study never used condoms, and $43 \%$ did so on an intermittent basis. In these groups, valacyclovir use prevented one case of genital herpes for every 40-60 couples receiving it. By contrast, if condoms were always used, the pharmacologic intervention prevented one new case of genital herpes for every 140 couples recieving it.

In reviewing the current recommendations to reduce the risk of transmission of genital herpes, four key strategies are identified [101]:

- Disclosure of genital herpes status before sexual contact

- Abstinence during outbreaks

- Consistent condom use [10]

- Pharmacologic intervention [9]

The issue of disclosure needs to be part of the public health education mandate and is a question that needs to be addressed with relevant authorities with some degree of urgency. There is no downside to this and every effort should be made to implement it as soon and as widely as possible. Individual physicians may also be engaged in the delivery of this message to their patients. Although most of the new cases of genital herpes occur while the infected host is asymptomatic, it is clear that the period of time surrounding a clinical outbreak is one of intense viral shedding, which carries an increased risk of transmission. Avoiding sexual contact during this time may be quite important and should be emphasized on a personal basis by physicians treating this condition.

Ensuring consistent condom use is easier said than done, but should nonetheless be attempted for the benefit of the $25-35 \%$ of individuals who are prepared to receive and act upon this information [9]. As a first step, pharmacologic intervention should then be considered in the $65-75 \%$ of individuals who are unable or unwilling to act upon this recommendation [9]. But if this is the only criterion we apply for intervention, we will still be left resorting to valacyclovir treatment in as many as one in eight adults. Can we begin to narrow it down a bit more to make the task more legitimate and realistic?

Let us consider two specific groups: females of child bearing potential, and monogamous heterosexual couples discordant for genital herpes. In the former group, a potentially lethal consequence of genital herpes infection would be its transmission to the neonate. The highest risk of this occurrence would be in the setting of a primary infection in the mother during pregnancy, especially if this occurs in the third trimester [11,12]. A highly effective intervention to potentially eradicate this problem is to incorporate specific testing for genital herpes for pregnant women and their partners in the first trimester medical evaluation. This can include a careful medical history supplemented by serologic testing for genital herpes to definitively establish which of the partners is infected. This is greatly facilitated by the availability of rapid, inexpensive type-specific herpes antibody assays. Some have suggested that these be incorporated into routine blood tests performed early in pregnancy. In situations where the male partner is infected and the female is not, a strong recommendation could be made to place the male partner on valacyclovir to prevent infection of the woman during the course of the pregnancy.

$\therefore$ most of the new cases of genital

herpes occur while the infected host is

asymptomatic ... the period of time surrounding a clinical outbreak is one of intense viral shedding, which carries an increased risk of transmission.'

A second group to consider for this intervention might be the discordant, monogamous couples who were the subject of the Corey study [9]. As such couples seek medical care, establishment of their genital herpes infection status could become routine, and pharmacologic intervention as transmission reduction therapy could be offered to discordant couples at highest risk. This would include situations where the male partner is infected (especially if the primary infection is recent) as well as in new sexual relationships. On the latter point, it would be especially relevant to include men and women in the fifth or sixth decade of life who, after having had a single long-term partner, suddenly find themselves with a new one, at an age where the cumulative prevalence of genital herpes is at its highest, approaching $40 \%$ in women [13]. If valacyclovir therapy is initiated, there are no data to guide us as to its optimal duration (i.e., whether this should be for the lifetime of the individual, or at least while sexual contact is ongoing). A more realistic first step would be to consider it for the first 2 years of a new relationship, while the risk of transmission is at its highest. 
It is quite clear that the burden of this intervention will lie on the shoulders of family physicians (or their equivalent). If they are to take on this task successfully, we must first ensure that they are given the facts that underlie the development of any recommendations we would make, to allow them to implement them with conviction. This will have to be tailored on an individual basis, but will have to include continuing education sessions as well as access to appropriate written materials and handouts to consolidate knowledge. We will also have to ensure access to type-specific herpes serology testing to support the effort. Finally, as we will be asking very busy individuals to add another task to their daily work schedule, we must give them the tools to be able to deal with infected individuals and their partners in a time effective manner. This must include the development of practical algorithms to streamline office visits, patient education materials and access to counseling services that many infected patients require.

Genital herpes is a pandemic that has developed in our midst while we, as a healthcare community, were occupied (or distracted) dealing with epidemics or pandemics with more evident clinical consequences (such as HIV infection) or that capture the public's imagination (such as the risk of avian influenza). Not only is genital herpes here to stay, it may actually be interacting with other pandemic pathogens (such as HIV) to worsen their impact and transmissibility [14]. Awareness of these facts is key, but it will be important to empower primary healthcare providers to take charge of the problem and give them the tools to intervene to begin to make a difference.

\section{Financial \& competing interests disclosure}

The author has received research support, consulting fees and honoraria from Glaxo Smith Kline and Novartis, manufacturers of medications used for the treatment of genital herpes.

The author has no other relevant affiliations or financial involvement with any organization or entity with a financial interest in or financial conflict with the subject matter or materials discussed in the manuscript apart from those disclosed.

No writing assistance was utilized in the production of this manuscript.

\section{Bibliography}

1. $\mathrm{XuF}$ et al:: Trends in herpes simplex virus type 1 and type 2 seroprevalence in the United States. JAMA 296, 964-973 (2006).

2. Armstrong GL, Schillinger J, Markowitz L et al: : Incidence of herpes simplex virus type 2 infection in the United States. Am. J. Epidemiol. 153, 912-920 (2001).

3. Patrick DM, Dawar M, Cook DA, Krajden M, Ng HC, Rekart ML: Antenatal seroprevalence of herpes simplex virus type 2 (HSV-2) in Canadian women: HSV-2 prevalence increases throughout the reproductive years. Sex. Transm. Dis. 28, 424-428 (2001).

4. Fleming DT, McGuillan GM, Johnson RE et al.: Herpes simplex virus type 2 in the United States, 1976 to 1994. N. Engl. J. Med. 337, 1105-1111 (1997).

5. Mertz GJ, Benedetti J, Ashley R et al:: Risk factors for the sexual transmission of genital herpes. Ann. Intern. Med. 116, 197-202 (1992).

6. Wald A, Zeh J, Selke S et al:: Virologic characteristics of subclinical and symptomatic genital herpes infections. N. Engl. J. Med. 333, 770-775 (1995).
7. Wald A, Zeh J. Selke S et al:: Genital shedding of herpes simplex virus among men. J. Infect Dis. 186(Suppl.1), S34-S39 (2002).

8. Wald A, Zeh J, Selke S et al:: Reactivation of genital herpes simplex type 2 infection in asymptomatic seropositive persons. N. Engl. J. Med. 342, 844-850 (2000).

9. Corey L, Wald A, Patel R et al.: Once-daily valacyclovir to reduce the risk of transmission of genital herpes. N. Engl. J. Med. 350, 11-20 (2004).

10. Wald, AM, Langenberg AG, Link K et al: : Effect of condoms on reducing the transmission of herpes simplex virus type 2 from men to women. JAMA 285, 3100-3106 (2001).

11. Brown ZA, Wald A, Morrow RA, Selke S, Zeh J, Corey L: Effect of serologic status and cesarean delivery on transmission rates of herpes simplex virus from mother to infant. JAMA 289, 203-209 (2003).

12. Brown ZA, Selke $S$, Zeh J et al: : The acquisition of herpes simplex virus during pregnancy. N. Engl. J. Med. 337, 509-515 (1997).
13. Leone P et al:: Seroprevalence of herpes simplex virus- 2 in suburban primary care offices in the United States. Sex. Transm. Dis. 31, 311-316 (2004).

14. Wald A, Link K: Risk of human immunodeficiency virus infection in herpes simplex virus type 2 -seropositive persons: a meta-analysis. J. Infect Dis. 185, 45-52 (2002).

\section{Website}

101. Aoki FY: Canadian Guidelines on Sexually Transmitted Infections 2006 Edition: Genital Herpes Simplex Virus (HSV) Infections. Public Health Agency of Canada, Ottowa, ON, Canada (2006) http://phac-aspc.gc.ca/stdmts/sti_2006/pdf_2006_e.html.

\section{Affiliation}

- Brian Conway, MD, FRCPC, Associate Professor University of British Columbia, Downtown ID Clinic, 201-1200 Burrard Street, Vancouver, BC V6Z2C7, Canada

Tel.: +1604642 6429;

Fax: +16046426419 ;

bconway@interchange.ubc.ca 